hep-ph/0302176

OSU-HEP-03-4

February, 2003

\title{
Unification of Gauge, Higgs and Matter in Extra Dimensions
}

\author{
Ilia Gogoladze ${ }^{1}$, Yukihiro Mimura ${ }^{2}$ and S. Nandi ${ }^{3}$ \\ Physics Department, Oklahoma State University, \\ Stillwater, OK 74078
}

\begin{abstract}
We consider the unification of gauge, Higgs as well as the matter fields in a $6 \mathrm{D} N=2$ supersymmetric $S U(8)$ gauge theory. The gauge symmetry $S U(8)$ is broken down to $S U(4) \times$ $S U(2)_{L} \times S U(2)_{R} \times U(1)^{2}$ in $4 \mathrm{D}$ through $T^{2} / Z_{6}$ orbifold compactification, and the theory is reduced to $4 \mathrm{D} N=1$ supersymmetric Pati-Salam model. The electroweak Higgs fields as well as the third family of fermions are unified in the $6 \mathrm{D} N=2$ gauge multiplet. The $6 \mathrm{D}$ bulk gauge interaction provides both gauge and Yukawa interactions for the third family predicting $\alpha_{1}=\alpha_{2}=\alpha_{3}=\alpha_{t}=\alpha_{b}=\alpha_{\tau}$ at the unification scale, in good agreement with experiment. Incorporation of the first and second family as well as other orbifolds are also briefly discussed.
\end{abstract}

\footnotetext{
${ }^{1}$ On a leave of absence from: Andronikashvili Institute of Physics, GAS, 380077, Tbilisi, Georgia. email: ilia@hep.phy.okstate.edu

2 email: mimura@hep.phy.okstate.edu

${ }^{3}$ email: shaown@okstate.edu
} 


\section{Introduction}

Recent topics of the theories in higher dimensions give us a lot of interesting phenomenological pictures. One of the most attractive motivations of extension of dimensions is that the variety of particles in Nature can be understood by means of a geometrical language. For example, gauge fields with the extra dimensional components behave as scalar fields in 4 dimension. Since masses of the gauge bosons are prohibited by gauge invariance, the scalar field originated from the gauge bosons can be a good candidate for the low energy Higgs fields which break electroweak symmetry. That leads to the idea of the gauge-Higgs unification in the higher dimensional theories [1, 2, 3].

We consider that the extra dimensions are compactified in an orbifold. In an orbifold space, we can impose transformation properties to the fields and the symmetries can be broken [2, 4. Recently, a great deal of works has been done on the gauge symmetry breaking using the orbifold compactification, and these lead to many attractive features of the unified gauge theories in higher dimensions [5, 6]. Using the orbifold transformation properties, we can project out unwanted fields such as colored Higgs triplets in the grand unified theories [5]. In such a progress of the higher dimensional unified theories, many people have revisited the idea of gauge-Higgs unification [7, 8, 9, 10, 11]. In the higher dimensional supersymmetric theories, the gauge multiplet contains both vector multiplet and chiral supermultiplets in 4D. Assigning the different transformation property between vector multiplet and chiral supermultiplets, we can make vector multiplet massless but chiral supermultiplets heavy, which means the supersymmetry is broken. If we break gauge symmetry through boundary condition simultaneously, a part of the chiral supermultiplets can have a zero mode which remains massless in the low energy. Then, we can identify such a supermultiplet with the low energy Higgs field. This idea is featured in the $6 \mathrm{D} N=2$ supersymmetric theories [8], and more recently in the 5D $N=1$ supersymmetric theories [9, 10, 11]. The Ref. [9] emphasizes an interesting possibility that the gauge and Yukawa coupling constants have the same origin. Since the Yukawa interactions arise from the gauge interaction in the 5D lagrangian, those two coupling constants are "unified" in the 5D theory. In the Ref. [9], the authors considered the theory of $S U(3)_{w}$ and $S U(6)$ as an example of this scenario. The gauge-Higgs unification in larger gauge group such as $E_{6}, E_{7}$ and $E_{8}$ has also been studied [10] In Ref. [11, the authors consider the gauge-Higgs unification in the $S U(4)_{w}$ and $S O(12)$, and suggest that the left-right symmetric breaking of the gauge symmetry gives an economical realization to the representation of the 
quarks and leptons.

Another interesting possibility of unified model in higher dimensions is that quarks and leptons can be unified in the gauge multiplet. Three families of quarks and leptons can be contained in the adjoint representations in large gauge groups, such as $E_{7}$ and $E_{8}$. The matters in the adjoint representation are always vector-like, but one can project out the vector-like partner by $Z_{3}$ transformation properties [12]. This encourages us to consider that the gauge and matter (quarks and leptons) unification in higher dimensional models. In other context, we can consider the origin of the three families as chiral superfields in gauge multiplet [13] since the gauge multiplet in the $6 \mathrm{D} N=2$ supersymmetry contains three $N=1$ chiral superfields in $4 \mathrm{D}$.

In this paper, we consider the possibility of unifying gauge, matter and Higgs fields in one supersymmetric gauge multiplet in higher dimensions, as well as the unification of the gauge and Yukawa couplings. As a simple example, we construct a $6 \mathrm{D} N=2$ supersymmetric $S U(8)$ unified model where such an unification is achieved. The gauge symmetry $S U(8)$ is broken down to $S U(4) \times S U(2)_{L} \times S U(2)_{R} \times U(1)^{2}$ in $4 \mathrm{D}$ through $T^{2} / Z_{6}$ orbifold compactification, and the theory is reduced to $4 \mathrm{D} N=1$ supersymmetric Pati-Salam model [14]. The electroweak Higgs fields and standard model fermions for the 3rd family can be unified with the gauge bosons in the $6 \mathrm{D}$ gauge multiplets. The $6 \mathrm{D}$ bulk gauge interaction produces Yukawa interactions, which give masses to the quarks and leptons by Higgs mechanism, and gives gauge-Yukawa unification. The numerical agreement of this gauge-Yukawa unification prediction for all the gauge and the third family Yukawa couplings is good.

Our paper is organized as follows: In section 2, we construct our supersymmetric $S U(8)$ model in 6D with gauge, Higgs and matter unification and show how orbifold compactification leads to Pati-Salam model in 4D. Numerical results for gauge and Yukawa coupling unification are shown in section 3. Section 4 contains our conclusions and discussions.

\section{The Model}

We consider the $6 \mathrm{D}$ gauge theory with $N=2$ supersymmetry. The two extra dimensions are compactified by the orbifold $T^{2} / Z_{n}$ [15]. The $N=2$ supersymmetry in 6D corresponds to $N=4$ supersymmetry in $4 \mathrm{D}$, thus only the gauge multiplet can be introduced in the bulk. In terms of $4 \mathrm{D} N=1$ language, the gauge multiplet contains vector multiplet $V\left(A_{\mu}, \lambda\right)$ and three chiral multiplets $\Sigma, \Phi$ and $\Phi^{c}$ in the adjoint representation of the gauge group. The fifth 
and sixth components of the gauge fields, $A_{5}$ and $A_{6}$, are contained in the lowest component of $\Sigma$, i.e. $\left.\Sigma\right|_{\theta=\bar{\theta}=0}=\left(A_{6}+i A_{5}\right) / \sqrt{2}$.

The bulk action, written in the $4 \mathrm{D} N=1$ language and in the Wess-Zumino gauge, is given by [16]

$$
\begin{aligned}
S= & \int d^{6} x\left\{\operatorname{Tr}\left[\int d^{2} \theta\left(\frac{1}{4 k g^{2}} W^{\alpha} W_{\alpha}+\frac{1}{k g^{2}}\left(\Phi^{c} \partial \Phi-\sqrt{2} \Sigma\left[\Phi, \Phi^{c}\right]\right)\right)+\text { h.c. }\right]\right. \\
& +\int d^{4} \theta \frac{1}{k g^{2}} \operatorname{Tr}\left[\left(\frac{1}{\sqrt{2}} \partial^{\dagger}+\Sigma^{\dagger}\right) e^{-2 V}\left(-\frac{1}{\sqrt{2}} \partial+\Sigma\right) e^{2 V}+\frac{1}{4} \partial^{\dagger} e^{-2 V} \partial e^{2 V}\right] \\
& \left.+\int d^{4} \theta \frac{1}{k g^{2}} \operatorname{Tr}\left[\Phi^{\dagger} e^{-2 V} \Phi e^{2 V}+\Phi^{c \dagger} e^{-2 V} \Phi^{c} e^{2 V}\right]\right\},
\end{aligned}
$$

where $k$ is the normalization of the group generator, $\operatorname{Tr} T^{a} T^{b}=k \delta^{a b}$ (we take $k=1 / 2$ ), $\partial$ is defined as $\partial=\partial_{5}-i \partial_{6}$, and $W_{\alpha}$ is defined as $W_{\alpha}=-\frac{1}{8} \bar{D}^{2}\left(e^{-2 V} D_{\alpha} e^{2 V}\right)$. The 6D gauge transformations are

$$
\begin{aligned}
e^{2 V} & \rightarrow e^{\Lambda} e^{2 V} e^{\Lambda^{\dagger}}, \quad \Sigma \rightarrow e^{\Lambda}\left(\Sigma-\frac{1}{\sqrt{2}} \partial\right) e^{-\Lambda}, \\
\Phi & \rightarrow e^{\Lambda} \Phi e^{-\Lambda}, \quad \Phi^{c} \rightarrow e^{\Lambda} \Phi^{c} e^{-\Lambda} .
\end{aligned}
$$

The $T^{2} / Z_{n}$ orbifold is constructed by identifying the complex coordinate $z$ of the extra dimensions under $Z_{n}: z \rightarrow \omega z$, where $\omega^{n}=1$. The number $n$ is restricted to be $n=2,3,4,6$. We can impose the transformation property of the gauge multiplet as

$$
\begin{aligned}
V\left(x^{\mu}, \omega z, \bar{\omega} \bar{z}\right) & =R V\left(x^{\mu}, z, \bar{z}\right) R^{-1}, \\
\Sigma\left(x^{\mu}, \omega z, \bar{\omega} \bar{z}\right) & =\bar{\omega} R \Sigma\left(x^{\mu}, z, \bar{z}\right) R^{-1} \\
\Phi\left(x^{\mu}, \omega z, \bar{\omega} \bar{z}\right) & =\omega^{l} R \Phi\left(x^{\mu}, z, \bar{z}\right) R^{-1} \\
\Phi^{c}\left(x^{\mu}, \omega z, \bar{\omega} \bar{z}\right) & =\omega^{m} R \Phi^{c}\left(x^{\mu}, z, \bar{z}\right) R^{-1}
\end{aligned}
$$

where $R$ is a unitary matrix and satisfies that $R^{n}$ is the identity matrix. Non-trivial $R$ breaks the gauge symmetry. Because of the lagrangian invariance in Eq.(11) under the transformations (447), we have a relation $l+m \equiv 1(\bmod n)$. In the case $n>2$, this transformation property breaks $N=4$ supersymmetry down to $N=1$ in $4 \mathrm{D}$.

Now we consider the $S U(8)$ gauge symmetry and $T^{2} / Z_{6}$ orbifold. We choose the $8 \times 8$ matrix $R$ as

$$
R=\operatorname{diag}\left(1,1,1,1, \omega^{5}, \omega^{5}, \omega^{2}, \omega^{2}\right) .
$$

With this choice, $S U(8)$ breaks down to $S U(4) \times S U(2)_{L} \times S U(2)_{R} \times U(1)^{2}$, and the theory is reduced to $4 \mathrm{D} N=1$ supersymmetric Pati-Salam model with two extra $U(1)$ symmetry. 
The $S U(8)$ adjoint representation $\mathbf{6 3}$ is decomposed under $S U(4) \times S U(2)_{L} \times S U(2)_{R} \times U(1)^{2}$ representations as

$$
\mathbf{6 3}=\left(\begin{array}{ccc}
(\mathbf{1 5}, \mathbf{1}, \mathbf{1})_{0,0} & (\mathbf{4}, \mathbf{2}, \mathbf{1})_{2,0} & (\mathbf{4}, \mathbf{1}, \mathbf{2})_{2,4} \\
(\overline{\mathbf{4}}, \mathbf{2}, \mathbf{1})_{-2,0} & (\mathbf{1}, \mathbf{3}, \mathbf{1})_{0,0} & (\mathbf{1}, \mathbf{2}, \mathbf{2})_{0,4} \\
(\overline{\mathbf{4}} \mathbf{1}, \mathbf{2})_{-2,-4} & (\mathbf{1}, \mathbf{2}, \mathbf{2})_{0,-4} & (\mathbf{1}, \mathbf{1}, \mathbf{3})_{0,0}
\end{array}\right)+(\mathbf{1}, \mathbf{1}, \mathbf{1})_{0,0}+(\mathbf{1}, \mathbf{1}, \mathbf{1})_{0,0},
$$

where the subscripts denote the charges under the $U(1)_{1} \times U(1)_{2}$ symmetry. The $Z_{6}$ transformation property for these respective decomposed representations of the vector multiplet $V$ and chiral multiplet $\Sigma$ is

$$
V:\left(\begin{array}{ccc}
1 & \omega & \omega^{4} \\
\omega^{5} & 1 & \omega^{3} \\
\omega^{2} & \omega^{3} & 1
\end{array}\right)+(1)+(1), \quad \Sigma:\left(\begin{array}{ccc}
\omega^{5} & 1 & \omega^{3} \\
\omega^{4} & \omega^{5} & \omega^{2} \\
\omega & \omega^{2} & \omega^{5}
\end{array}\right)+\left(\omega^{5}\right)+\left(\omega^{5}\right) .
$$

We see from (10) that the gauge fields for the unbroken symmetry group have massless modes in $V$, and $(\mathbf{4}, \mathbf{2}, \mathbf{1})_{2,0}$ component in $\Sigma$ has massless mode. Choosing $l=4$ and $m=3$, we obtain the transformation property for $\Phi$ and $\Phi^{c}$ as

$$
\Phi:\left(\begin{array}{ccc}
\omega^{4} & \omega^{5} & \omega^{2} \\
\omega^{3} & \omega^{4} & \omega \\
1 & \omega & \omega^{4}
\end{array}\right)+\left(\omega^{4}\right)+\left(\omega^{4}\right), \quad \Phi^{c}:\left(\begin{array}{ccc}
\omega^{3} & \omega^{4} & \omega \\
\omega^{2} & \omega^{3} & 1 \\
\omega^{5} & 1 & \omega^{3}
\end{array}\right)+\left(\omega^{3}\right)+\left(\omega^{3}\right)
$$

and thus $(\overline{\mathbf{4}}, \mathbf{1}, \mathbf{2})_{-2,-4}$ in $\Phi,(\mathbf{1}, \mathbf{2}, \mathbf{2})_{0,4}$ and $(\mathbf{1}, \mathbf{2}, \mathbf{2})_{0,-4}$ in $\Phi^{c}$ have massless modes in $4 \mathrm{D}$. We identify $(\mathbf{4}, \mathbf{2}, \mathbf{1})$ and $(\overline{4}, \mathbf{1}, \mathbf{2})$ as left- and right-handed quarks and leptons respectively in one family, and two bidoublets $(\mathbf{1}, \mathbf{2}, \mathbf{2})$ as Higgs fields which break electroweak symmetry. Then Higgs fields and one family of matter fields are unified with the gauge fields in the $6 \mathrm{D} N=2$ gauge multiplet. We denote those fields as

$$
\Psi_{L}:(\mathbf{4}, \mathbf{2}, \mathbf{1})_{2,0}, \quad \Psi_{R}:(\overline{4}, \mathbf{1}, \mathbf{2})_{-2,-4} \quad H_{1}:(\mathbf{1}, \mathbf{2}, \mathbf{2})_{0,4}, \quad H_{2}:(\mathbf{1}, \mathbf{2}, \mathbf{2})_{0,-4}
$$

We note briefly the other choices of $l, m$ and unitary matrix $R$. We may have three solutions of $l+m \equiv 1(\bmod 6),(l, m) \equiv(0,1),(5,2),(4,3)$ without loss of generality. (Exchanging of $l$ and $m$ gives identical sets of zero-modes.) Only the choice of $(l, m)=(4,3)$ leads to one family of matter as the zero-modes. With the different choice of $R$ from Eq.(8), contents of zero-modes will be different. We have chosen the unitary matrix $R$ so that both left- and right-handed matters are chiral, and at that time, we get two Higgs bidoublets as zero-modes. We have also chosen the left-handed matter is in the chiral multiplet $\Sigma$.

Since the three $N=1$ chiral multiplets $\Sigma, \Phi$ and $\Phi^{c}$ are in the gauge multiplets, those fields have gauge interactions with each other in 6D. This interaction term from Eq.(11) is

$$
S=\int d^{6} x\left[\int d^{2} \theta \frac{1}{k g^{2}} \operatorname{Tr}\left(-\sqrt{2} \Sigma\left[\Phi, \Phi^{c}\right]\right)+\text { h.c. }\right],
$$


includes Yukawa interaction

$$
S=\int d^{6} x \int d^{2} \theta y_{6} \Psi_{L} H_{1} \Psi_{R}+\text { h.c. }
$$

Taking into account the normalization factor of $\Psi_{L}, \Psi_{R}$ and $H_{1}$ in the kinetic term, we find that the six dimensional Yukawa coupling is equal to six dimensional gauge coupling, $y_{6}=g_{6}$. The corresponding four dimensional couplings are derived as the coordinates of extra dimensions are integrated out in the action. In the ideal situation, the four dimensional Yukawa and gauge coupling can be the same dimensionless number. The ideal situation is the following: 1) The brane-localized gauge and Yukawa interactions can be negligible. 2) The zero modes of fermions $\Psi_{L}$ and $\Psi_{R}$ are not localized at different points on the orbifold. 3) The four dimensional fields are not largely mixed with other brane-localized fields.

As for the first situation, the $4 \mathrm{D}$ gauge couplings can receive contributions from branelocalized gauge kinetic terms, such as $\delta(z) F_{\mu \nu}^{2}$. However, such contributions can be expected negligible if the volume of extra dimensions is large. Since the left-handed fermions $\Psi_{L}$ is obtained from $\Sigma$ which includes the gauge fields with fifth and sixth coordinates, the $\Psi_{L}$ transforms non-linearly under gauge transformation in Eq.(2). Thus, we cannot write the brane-localized Yukawa interaction. If we consider Wilson-line operator, we may write a gauge invariant 4D interaction, but again the contribution can be negligible if the volume of the extra dimensions is large. Next, we consider second ideal situation. If the zero modes of fermions are localized at different points, the 4D Yukawa couplings become proportional to the overlap of the zero modes wave functions. In general, vacuum expectation values of the singlet in the three chiral multiplets gives bulk masses of the fermions, and then the zero modes might be localized around the 3-brane. But if the vacuum expectation values are smaller than $1 / R$ ( $R$ is a radius of the extra dimensions), the contribution can be negligible. The situation 3 ) is just the usual things of the four dimensional model building. If the fields mix with some other fields and the mass eigenvalues are the linear combination of the mixed fields, the coupling for the mass eigenstates is the original couplings multiplied with the mixing angle. Thus, the mixing angle is small, the Yukawa coupling is almost same as the gauge couplings.

We identify the one family originating from gauge multiplet with the 3rd family, and 1st and 2nd family are brane-localized fields at 3-brane fixed point. Then the Yukawa couplings for 3rd family, $y_{t}, y_{b}$ and $y_{\tau}$, are unified to the gauge couplings at grand unified scale. We will see the numerical studies in Section 4. The Yukawa couplings of 1st and 2nd family are naturally smaller than the 3rd family couplings, since their values are suppressed by volume 
factor of the extra dimensions.

Since we project out the vector-like partners, the remaining fermion components Eq.(12) give gauge anomaly for the extra two $U(1)$ symmetry. Both two linear combinations of $U(1)$ symmetry have gauge anomaly, and Green-Schwarz mechanism [17] can cancel out only one linear combination. Thus we have to introduce other brane fields to cancel the anomaly. For example, if we introduce brane fields with appropriate $U(1)$ charge such as

$$
\Psi_{L}^{\prime}:(4,2,1)_{-2,0}, \quad \Psi_{R}^{\prime}:(\overline{4}, 1,2)_{2,4}
$$

all gauge anomaly is cancelled out. This can be an origin of other families. Since one linear combination of $U(1)$ can be anomalous by using Green-Schwarz mechanism, we can make hierarchical structure in the quark and lepton mass matrices by Froggatt-Nielsen like mechanism [18.

We have made a choice the left-handed matter is in the chiral multiplet $\Sigma$ and right-handed one is in the $\Phi$. Since the gauge transformations of $\Sigma$ and $\Phi$ in Eqs.(2) (3) are different, branelocalized 4D lagrangian is not left-right symmetric. The right-handed quark and lepton and Higgs fields are in the chiral multiplets $\Phi$ and $\Phi^{c}$ and their gauge transformations are linear, and thus we can introduce the brane-localized Yukawa coupling terms which give the CKM mixing angles. We can also introduce the brane-localized right-handed neutrino mass terms with the fields $(\mathbf{4}, \mathbf{1}, \mathbf{2})$ and/or $(\overline{4}, \mathbf{1}, \mathbf{2})$ in the chiral multiplet $\Phi^{c}$, and the neutrino masses become small through the seesaw mechanism. The vacuum expectation values of $(\mathbf{4}, \mathbf{1}, \mathbf{2})$ and/or $(\overline{4}, \mathbf{1}, 2)$ give right-handed Majorana neutrino masses and also break the Pati-Salam gauge symmetry down to the standard model gauge symmetry. We may also use the brane Higgs field $(\mathbf{1 0}, \mathbf{1}, \mathbf{3})$ to give the right-handed neutrinos masses and to break the Pati-Salam gauge symmetry.

In the Pati-Salam model, we need at least two Higgs multiplets, otherwise the down-type quark mass matrix is proportional to up-type quark mass matrix. In our model, we have two Higgs bidoublets. Requiring the Goergi-Jarskog relation [19] for 2nd family, we need another Higgs field such as $(\mathbf{1 5}, \mathbf{2}, \mathbf{2})$. For the gauge coupling unification, only one linear combination of Higgs bidoublet fields should remain at low energy. The mixing parameter can be controlled by using the extra $U(1)$ symmetries.

We comment that we can consider three family and Higgs unification in $T^{2} / Z^{3}$ orbifold if we take $l=m=2$ in Eqs. (6.7]) and

$$
R=\operatorname{diag}\left(1,1,1,1, \omega^{2}, \omega^{2}, \omega, \omega\right) .
$$


In this case, the origin of the number of family is the number of chiral multiplets in the $N=4$ gauge multiplet. In our model building, the Yukawa couplings arise from gauge interaction in Eq.(13). It is easy to see that the mass matrices for the quarks and leptons are anti-symmetric, and then one of the family is massless and other two families have same mass. We can modify this results by adding the brane-localized interactions, but it is difficult to split masses of 2 nd and 3rd families while preserving gauge and Yukawa coupling unification. Of course, we have to care about the $U(1)$ gauge anomaly and we have to introduce other fields to cancel the anomaly.

\section{Numerical Results of Gauge and Yukawa Unification}

As was mentioned in the previous section this model can realize gauge-Yukawa unification for third family quarks and lepton ${ }^{4}$. For realization of this possibility we assume that the compactification scale $\left(M_{U}\right)$ from $6 \mathrm{D}$ to $4 \mathrm{D}$ is the same scale where $S U(4) \times S U(2)_{L} \times S U(2)_{R} \times U(1)^{2}$ gauge symmetry are broken to Standard Model one, choosing appropriate Higgs superfields, which are localized in 4D. So below $M_{U}$ scale we have usual MSSM particle content with gauge-Yukawa unification condition for particles from third family

$$
\alpha_{1}=\alpha_{2}=\alpha_{3}=\alpha_{t}=\alpha_{b}=\alpha_{\tau}
$$

where $\alpha_{1}, \alpha_{2}$ and $\alpha_{3}$ corresponds hypercharge (with proper normalization), weak and strong interaction couplings. $\alpha_{t}, \alpha_{b}, \alpha_{\tau}$ are the top, bottom and tau Yukawa coupling respectively. We use the notation $y_{t, b, \tau}^{2} / 4 \pi \equiv \alpha_{t, b, \tau}$. Note that we neglect brane localized gauge kinetic term.

For numerical calculation of gauge and Yukawa coupling evolution we are using two-loop RG analysis [21, with conversion from $\overline{M S}$ scheme to dimensional reduction $(\overline{D R})$ one. We include the standard supersymmetric threshold correction at low energies, taken at a single scale $M_{S U S Y}=M_{Z}[22$.

Due to a crucial reduction of the number of the fundamental parameters from the gaugeYukawa coupling unification, we are lead immediately to a series of the very distinctive predictions (in absence of any large supersymmetric threshold corrections). Using the values of the electroweak parameters $\sin ^{2} \theta_{w}=0.2311 \pm 0.0001$ and $\alpha_{E M}=127.92 \pm 0.02$ at $M_{Z}$ scale [23], we can determine the unification scale and unified coupling constant. Then, evolving the

\footnotetext{
${ }^{4}$ The numerical calculation of gauge-Yukawa unification in a $4 \mathrm{D}$ model is demonstrated in the Ref. [20].
} 


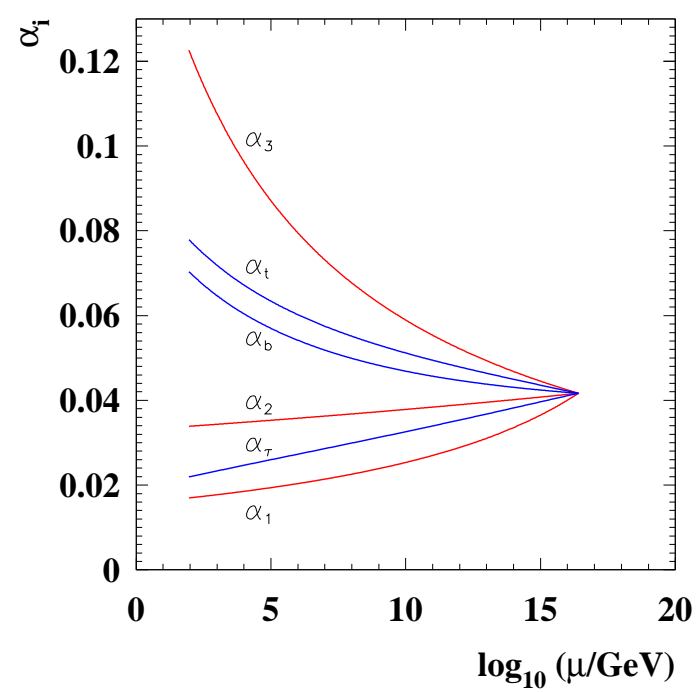

Figure 1: The unification of gauge $\left(\alpha_{1}, \alpha_{2}, \alpha_{3}\right)$ and Yukawa $\left(\alpha_{t}, \alpha_{b}, \alpha_{\tau}\right)$ couplings at SUSY GUT scale (red and blue lines respectively).

remaining couplings from the unification scale to the low energy, we predict

$$
\alpha_{3}\left(M_{Z}\right)=0.123, \quad m_{t}=178 \mathrm{GeV}, \quad \frac{m_{b}}{m_{\tau}}\left(M_{Z}\right)=1.77, \quad \tan \beta=51 .
$$

These are in good agreement with experimental data [23]. The small discrepancy for $\alpha_{3}$ (world average value is $\alpha_{3}=0.117 \pm 0.002$ [23] ) can be easily improved if we consider unification scale threshold of Higgs in the $(\mathbf{1 0}, \mathbf{1}, \mathbf{3})$ representation, which is brane localized field. After PatiSalam symmetry is broken to Standard Model by $(\mathbf{1 0}, \mathbf{1}, \mathbf{3})+(\overline{\mathbf{1 0}}, \mathbf{1}, \mathbf{3})$ in the particle spectrum we have $S U(3)_{c}$ color sextet $(\mathbf{6}+\overline{\mathbf{6}})$ Higgs field. Arranging for this fragment mass at the scale $10^{16} \mathrm{GeV}$ we can avoid discrepancy of $\alpha_{3}$ and we have $\alpha_{3}=0.117$. This threshold gives the

correction to the top quark mass to the right direction as unification constant increase from this correction and $m_{t}=177 \mathrm{GeV}$. In Figure 1, unification of the gauge-Yukawa coupling is demonstrated.

\section{Conclusion and Discussion}

The idea that the Higgs and/or the matter are unified in the higher dimensional supersymmetric gauge multiplet leads us naturally to the world in which the standard gauge group in $4 \mathrm{D}$ is unified in grand unified gauge group in higher dimensions.

We have considered the $6 \mathrm{D} N=2$ supersymmetric $S U(8)$ gauge theory in the orbifold $T^{2} / Z_{6}$. The gauge group $S U(8)$ is broken down by $Z_{6}$ transformation property, and the model 
is reduced to $N=1$ supersymmetric Pati-Salam model $S U(4) \times S U(2)_{L} \times S U(2)_{R}$ with two extra $U(1)$ symmetries in 4D. In this model, two bidoublet Higgs fields and one family of quarks and leptons are unified in the gauge sector in the 6D $N=2$ gauge supermultiplet. The vector-like partners of the quarks and leptons are projected out by $Z_{6}$ transformation property. The explicit mass terms of the Higgs fields are prohibited by the gauge invariance, and the $N=1$ supersymmetry preserve the masses of the Higgs fields to the electroweak scale. Furthermore, because of the unification of Higgs and matter in gauge multiplet, the 4D Yukawa interactions, which we need in order to give masses to the fermions by Higgs mechanism, arise from gauge interaction in 6D lagrangian. This is the most interesting feature of this model. The one family of quarks and leptons in the gauge multiplet can be identified to the 3rd family, and then we meet an attractive possibility that 3rd family Yukawa couplings can be unified to the gauge coupling at grand unified scale. The numerical results of the renormalization group flow of the Yukawa couplings have a good agreement with low energy data of the top quark mass and bottom-tau mass ratio with large $\tan \beta$.

Since one cannot put the additional bulk fields in $6 \mathrm{D} N=2$ supersymmetric theory, 1st and 2nd families must be brane-localized fields, and the Yukawa interactions for 1st and 2nd families are introduced at the 3-brane. Thus, the smallness of the masses of the 1st and 2nd families can be understood as the volume suppression of the Yukawa couplings. Since both extra $U(1)$ symmetries give gauge anomalies, we have to introduce additional fields to cancel them. The additional fields can be identified to another family. The extra $U(1)$ symmetry can be used to construct a hierarchical structure of the mass matrices.

It is interesting that the Yukawa interaction for 3rd family originates from $6 \mathrm{D}$ gauge interaction, whereas we cannot predict the Yukawa couplings for 1st and 2nd families which are brane fields. It is well-known that bottom-tau ratio is successful in the usual $S U(5)$ relation. In order to realize $m_{s} / m_{\mu}$, however, we have to introduce $(\mathbf{1 5}, \mathbf{2}, \mathbf{2})$ Higgs, which is included in 126 or 120 in $S O(10)$ language. Then, a question arises: Why 3rd family doesn't have Yukawa couplings with such $(\mathbf{1 5}, \mathbf{2}, \mathbf{2})$ Higgs field? We can answer the question in the context of our gauge-Higgs unification scenario. That is because $(\mathbf{1 5}, \mathbf{2}, \mathbf{2})$ Higgs is not included in the gauge multiplet and this Higgs must be brane-localized fields. The 3rd family Yukawa interactions with $(\mathbf{1 5}, \mathbf{2}, \mathbf{2})$ Higgs are suppressed by volume factor of extra dimensions. Thus, we can realize the unification of the bottom-tau Yukawa couplings.

We have used torus $T^{2}$ as the extra space manifold in this paper. Zero-modes of matter can be chiral through the $Z_{6}$ projection. At the same time, we can have two Higgs bidoublets 
as zero-modes. One can consider other manifolds, such as disc $D^{2}$ or annulus $A^{2}$. In that case, we can take $Z_{n}$ transformation with arbitrary number of $n$. For example, we can consider $D^{2} / Z_{9}$ orbifold and zero-modes of matter can be chiral through $Z_{9}$ projection. In this case, we can have one family and only one bidoublet of the Higgs.

We can also consider other unified gauge groups. It seems that $S O(12)$ is the smallest rank gauge group to unify the Higgs and the matter in gauge multiplet. The $S O(12)$ can be broken down to $S U(5) \times U(1)^{2}$ by $T^{2} / Z_{6}$ orbifold compactification. We can choose the

$Z_{6}$ projection so that $\mathbf{1 0}_{2,0}, \overline{\mathbf{5}}_{-2,2}, \mathbf{5}_{0,2}$ and $\overline{\mathbf{5}}_{0,-2}$ have zero modes. Thus the gauge multiplet contains Higgs fields and one family of matter. However, 6D gauge interaction in Eq.(13) doesn't include the top quark Yukawa interaction. Actually, the $U(1)$ symmetry doesn't match in the interaction term $10 \cdot \mathbf{1 0} \cdot \mathbf{5}$. Another example of gauge group is $E_{7}$. $E_{7}$ can be broken down to $E_{7} \rightarrow S O(10) \times U(1)^{2}$ by orbifold compactification. Since $S U(8)$ is one of the regular maximal subgroup of $E_{7}$, our model which we suggest in this paper can be one of the breaking pattern of the $E_{7}$.

Finally the idea of gauge, Higgs and matter unification is very novel. The fact that this can be semirealistically achieved in higher dimensional supersymmetric theories is very intriguing, and leads to yet another motivation for supersymmetry.

\section{Acknowledgments}

We thank K.S. Babu, C. Macesanu, J. Lykken and S. Raby for useful discussions. This work was supported in part by US DOE Grants \# DE-FG030-98ER-41076 and DE-FG-02-01ER45684.

\section{References}

[1] N. S. Manton, Nucl. Phys. B 158, 141 (1979);

D. B. Fairlie, J. Phys. G 5, L55 (1979); Phys. Lett. B 82, 97 (1979);

P. Forgacs and N. S. Manton, Commun. Math. Phys. 72, 15 (1980);

G. Chapline and R. Slansky, Nucl. Phys. B 209, 461 (1982);

D. Kapetanakis and G. Zoupanos, Phys. Rept. 219, 1 (1992). 
[2] Y. Hosotani, Phys. Lett. B 126, 309 (1983); Phys. Lett. B 129, 193 (1983); Phys. Rev. D 29, 731 (1984); Annals Phys. 190, 233 (1989).

[3] L. J. Hall, H. Murayama and Y. Nomura, Nucl. Phys. B 645, 85 (2002) hep-th/0107245; R. Dermisek, S. Raby and S. Nandi, Nucl. Phys. B 641, 327 (2002) hep-th/0205122].

[4] J. Scherk and J. H. Schwarz, Phys. Lett. B 82, 60 (1979); Nucl. Phys. B 153, 61 (1979); E. Witten, Nucl. Phys. B 258, 75 (1985); P. Candelas, G. T. Horowitz, A. Strominger and E. Witten, Nucl. Phys. B 258, 46 (1985); L. Dixon, J. Harvey, C. Vafa and E. Witten, Nucl. Phys. B 261, 651 (1985).

[5] Y. Kawamura, Prog. Theor. Phys. 103, 613 (2000) hep-ph/9902423; Prog. Theor. Phys. 105, 999 (2001) hep-ph/0012125; Prog. Theor. Phys. 105, 691 (2001) hep-ph/0012352.

[6] see for example

G. Altarelli and F. Feruglio, Phys. Lett. B 511, 257 (2001) hep-ph/0102301;

A. B. Kobakhidze, Phys. Lett. B 514, 131 (2001) hep-ph/0102323;

L. J. Hall and Y. Nomura, Phys. Rev. D 64, 055003 (2001) hep-ph/0103125; Phys. Rev. D 66, 075004 (2002) hep-ph/0205067;

A. Hebecker and J. March-Russel, Nucl. Phys. B613, 3 (2001) hep-ph/0106166; Nucl. Phys. B 625, 128 (2002) hep-ph/0107039;

T. j. Li, Phys. Lett. B 520, 377 (2001) hep-th/0107136; Nucl. Phys. B 619, 75 (2001) hep-ph/0108120;

C. Csaki, G. D. Kribs and J. Terning, Phys. Rev. D 65, 015004 (2002) hep-ph/0107266;

N. Maru, Phys. Lett. B 522, 117 (2001) hep-ph/0108002;

L. J. Hall, Y. Nomura, T. Okui and D. R. Smith, Phys. Rev. D 65, 035008 (2002) hep-ph/0108071;

R. Dermisek and A. Mafi, Phys. Rev. D 65, 055002 (2002) hep-ph/0108139;

T. Watari and T. Yanagida, Phys. Lett. B 519, 164 (2001) hep-ph/0108152;

Y. Mimura and S. Nandi, Phys. Lett. B 538, 406 (2002) hep-ph/0203126; 
F. Paccetti Correia, M. G. Schmidt and Z. Tavartkiladze, Nucl. Phys. B 649, 39 (2003) hep-ph/0204080; Phys. Lett. B 545, 153 (2002) hep-ph/0206307;

R. N. Mohapatra and A. Perez-Lorenzana, Phys. Rev. D 66, 035005 (2002) hep-ph/0205347;

N. Haba and Y. Shimizu, hep-ph/0210146; hep-ph/0212384;

I. Gogoladze, Y. Mimura and S. Nandi, Phys. Lett. B 554, 81 (2003) hep-ph/0210320;

J. Jiang, T. j. Li and W. Liao, hep-ph/0210436;

R. Kitano and T. Li, hep-ph/0302073.

[7] I. Antoniadis, Phys. Lett. B 246, 377 (1990);

N. V. Krasnikov, Phys. Lett. B 273, 246 (1991);

I. Antoniadis and K. Benakli, Phys. Lett. B 326, 69 (1994) hep-th/9310151;

H. Hatanaka, T. Inami and C. S. Lim, Mod. Phys. Lett. A 13, 2601 (1998) hep-th/9805067;

G. R. Dvali, S. Randjbar-Daemi and R. Tabbash, Phys. Rev. D 65, 064021 (2002) hep-ph/0102307;

N. Arkani-Hamed, A. G. Cohen and H. Georgi, Phys. Lett. B 513, 232 (2001) hep-ph/0105239;

I. Antoniadis, K. Benakli and M. Quiros, New J. Phys. 3, 20 (2001) hep-th/0108005;

C. Csaki, C. Grojean and H. Murayama, hep-ph/0210133.

[8] L. J. Hall, Y. Nomura and D. R. Smith, Nucl. Phys. B 639, 307 (2002) hep-ph/0107331.

[9] G. Burdman and Y. Nomura, hep-ph/0210257.

[10] N. Haba and Y. Shimizu, hep-ph/0212166.

[11] Q. Shafi and Z. Tavartkiladze, Phys. Rev. D 66, 115002 (2002);

I. Gogoladze, Y. Mimura and S. Nandi, Phys. Lett. B 560, 204 (2003) hep-ph/0301014.

[12] K. S. Babu, S. M. Barr and B. s. Kyae, Phys. Rev. D 65, 115008 (2002) hep-ph/0202178.

[13] T. Watari and T. Yanagida, Phys. Lett. B 532, 252 (2002) hep-ph/0201086; Phys. Lett. B 544, 167 (2002) hep-ph/0205090]. 
[14] J. C. Pati and A. Salam, Phys. Rev. D 10, 275 (1974).

[15] T. j. Li, Eur. Phys. J. C 24, 595 (2002) hep-th/0110065); Nucl. Phys. B 633, 83 (2002) hep-th/0112255;

C. S. Huang, J. Jiang, T. j. Li and W. Liao, Phys. Lett. B 530, 218 (2002) hep-th/0112046.

[16] N. Arkani-Hamed, T. Gregoire and J. Wacker, JHEP 0203, 055 (2002) hep-th/0101233.

[17] M. B. Green and J. H. Schwarz, Phys. Lett. B 149, 117 (1984).

[18] C. D. Froggatt and H. B. Nielsen, Nucl. Phys. B 147, 277 (1979).

[19] H. Georgi and C. Jarlskog, Phys. Lett. B 86, 297 (1979).

[20] J. L. Chkareuli and I. G. Gogoladze, Phys. Rev. D 58, 055011 (1998) hep-ph/9803335.

[21] M.B. Einhorn, D.R.T. Jones, Nucl. Phys. B 196 (1982) 475; W. Marciano, G. Senjanović, Phys. Rev. D 25 (1982) 3092; V. Barger, M.S. Berger and P. Ohmann, Phys. Rev D 47, 1093 (1993); S.P. Martin and M.T. Vaughn, Phys. Rev. D 50, 2282 (1994).

[22] P. Langacker and N. Polonsky, Phys. Rev. D 47, 4028 (1993); M. Carena, S. Pokorsky and C.E.M. Wagner, Nucl. Phys. B 406, 59 (1993).

[23] Particle Data Group, K. Hagiwara et. al, Phys. Rev. D 66, (2002) 010001-173. 